\title{
PIPERACILLIN (T-1220), A NEW SEMISYNTHETIC PENICILLIN: IN VITRO ANTIMICROBIAL ACTIVITY COMPARISON WITH CARBENICILLIN, TICARCILLIN, AMPICILLIN, CEPHALOTHIN, CEFAMANDOLE AND CEFOXITIN
}

\author{
R. N. JONES \\ Clinical Microbiology Laboratory, Kaiser Foundation Hospital Laboratories \\ Clackamas, Oregon 97015, U.S.A. \\ C. THORNSBERRY
}

Antimicrobic Investigation Section, Center for Disease Control, Atlanta, Georgia 30333, U.S.A.

\author{
A. L. BARRY
}

Clinical Microbiology Laboratories, University of California (Davis)

Sacramento Medical Center, Sacramento, California 95817, U.S.A.

P. C. FUCHS

Department of Pathology, St. Vincent Hospital and Medical Center, Portland, Oregon 97225, U.S.A.

\section{T. L. GavaN}

Department of Microbiology, The Cleveland Clinic, Cleveland, Ohio 44106, U.S.A.

\author{
and E. H. GerLach
}

Microbiology Laboratory, St. Francis Hospital, Wichita, Kansas 67214, U.S.A.

(Received for publication October 3, 1977)

\begin{abstract}
Piperacillin (T-1220) is a new semisynthetic penicillin with an unusually broad spectrum of antimicrobial activity. In vitro comparisons of this drug with 6 other beta-lactam antimicrobics (ticarcillin, carbenicillin, ampicillin, cephalothin, cefamandole and cefoxitin) were conducted. These included minimal inhibitory concentrations (MIC) against 394 bacterial isolates, the minimal lethal concentrations (MLC) against 79 of those, as well as the effect of inoculum size on the MIC and MLC of the drugs. Piperacillin had significantly greater activity than did the other penicillins against Pseudomonas species and Klebsiella pneumoniae. Against $P$. aeruginosa piperacillin was 8 - and 16 -fold more active than ticarcillin and carbenicillin, respectively. The MLC of piperacillin rarely differed from the MIC by more than one $\log _{2}$ dilutions except against $P$. aeruginosa in which the MLC was 4-fold greater or more than the MIC of $45 \%$ of isolates tested. Ticarcillin, carbenicillin and cefoxitin showed minimal inoculum size effects. Cefamandole results showed the greatest inoculum size variation with $55 \%$ and $37 \%$ of isolates showing an 8-fold increase in MIC and MLC respectively by increasing inoculum from $10^{5}$ to $10^{7} \mathrm{CFU} / \mathrm{ml}$. Piperacillin was intermediately effected having $25 \%$ of strains $>8$-fold increase in MIC.
\end{abstract}

Piperacillin, sodium 6-[D(-)- $\alpha$ (4-ethyl-2,3-dioxo-1-piperazinylcarbonylamino)- $\alpha$ - phenylacetamido]<smiles>CCN1CCN(C(=O)NC(C(=O)NC2C(=O)N3C(=O)C2C3C(=O)NC)c2ccccc2)C(=O)C1(C)C</smiles>
penicillinate, is a new semisynthetic penicillin derived from aminobenzyl-penicillin (Fig. 1). This compound formerly known as T-1220, has potent antibacterial activity against bacterial species previously refractory to such related compounds as carbenicillin and ticarcillin. In addition, favorable pharmacokinetics and infection studies have been reported in humans and in experimental animals (abstracts 349 and 350, Sixteenth Interscience 
Conference on Antimicrobial Agents and Chemotherapy, Chicago, 1976). This in vitro investigation directly compares the antimicrobial activity of piperacillin with that of related semisynthetic penicillins carbenicillin, ticarcillin, and ampicillin, plus three reference cephalosporins - cephamycins, cephalothin, cefamandole and cefoxitin.

\section{Materials and Methods}

Antibiotics:

The beta-lactam antimicrobic laboratory standard powders were supplied by the following pharmaceutical companies: Piperacillin from Lederle Laboratories, Pearl River, New York; cefamandole and cephalothin from Eli Lilly \& Company, Indianapolis, Indiana; cefoxitin from Merck Sharp \& Dohme, Rahway, New Jersey; carbenicillin and ticarcillin from Beecham Laboratories, Bristol, Tennessee; and ampicillin from Bristol Laboratories, Syracuse, New York.

\section{Organisms:}

A total of 394 bacterial isolates were contributed by the collaborating laboratories for this study. These include 149 strains of the Enterobacteriaceae, 160 strains of non-enterobacteriaceae gram-negative bacilli, and 85 strains of gram-positive cocci. They were further subdivided into the following genus and species categories: 29 Escherichia coli, 25 Klebsiella pneumoniae, 24 Enterobacter species, 27 indolepositive Proteus species, 24 Proteus mirabilis, 20 Serratia species, 108 Pseudomonas aeruginosa, 10 Pseudomonas cepacia, 6 Pseudomonas fluorescens-putida, 10 Pseudomonas maltophilia, 4 Pseudomonas stutzeri, 11 Acinetobacter anitratus, 11 Aeromonas hydrophila, 30 Staphylococcus aureus (10 methicillinresistant), 15 Streptococcus faecalis, 20 Streptococcus pneumoniae and 20 Streptococcus pyogenes.

Most of the isolates were tested in duplicate by two of the collaborating laboratories (Center for Disease Control and the Sacramento Medical Center) in a manner previously reported. ${ }^{1,2)}$ A third laboratory, Kaiser Foundation, also tested a more limited number (MLCs and inoculum size studies). No significant variation in MIC results were encountered between these participating laboratories.

Antimicrobic Susceptibility Testing:

Minimum inhibitory concentrations (MICs) were determined by the broth microdilution method. Mueller-Hinton broth was commercially dispensed in a single lot of plastic trays (Micro Media Systems, San Jose, California) and distributed to the testing laboratories. The trays were stored at $-60^{\circ} \mathrm{C}$ until inoculated. Prior to use the trays were thawed at room temperature (approximately $20 \sim$ 30 minutes) and inoculated with disposable inoculators delivering $5 \mu \mathrm{l}$ to each well.

At all three laboratories, a logarithmic phase broth culture was diluted to match the turbidity of a 0.5 MACFARLAND standard. The suspension was then diluted $1: 50$ in sterile water containing $0.02 \%$ Tween 80 and dispensed as described earlier. Final inoculum achieved was $1 \times 10^{5}$ colony forming units (CFU) per ml. For the testing of the fastidious streptococci including S. pyogenes and S. pneumoniae, the inoculum was standardized in MueLLer-Hinton broth containing $5 \%$ lysed rabbit blood and $0.1 \mathrm{ml}$ of this adjusted cell suspension was added to each microdilution well, giving a final concentration of $1 \times 10^{5} \mathrm{CFU} / \mathrm{ml}$.

The MIC was recorded as the lowest concentration totally inhibiting bacterial growth (clear well), after approximately 18 hours of incubation at $35^{\circ} \mathrm{C}$ in a forced air incubator. Occasionally, visible growth occurred in concentrations 1 2 wells above the MIC (the skipped-tube phenomenon).

Minimum lethal concentrations were determined for 79 organisms from seven genera by subculturing $5 \mu \mathrm{l}$ from each microdilution well. The $5 \mu \mathrm{l}$ subcultures were transferred to trypticase soy agar with $5 \%$ sheep blood. The subcultures were made with multiple inoculum replicator onto a $150 \times 100 \mathrm{~mm}$ plate. After 48 hours of incubation, the endpoints were read as the lowest concentration yielding no more than $0.1 \%$ survivors $(99.9 \%$ kill).

The effect of varying the inoculum concentrations on MIC-MLC endpoints was studied on 79 rapid growing facultative anaerobes. Trays were inoculated to achieve final concentrations of $10^{3}$, $10^{5}$, and $10^{7} \mathrm{CFU} / \mathrm{ml}$. MICs and MLCs were interpreted as described above. 
Results

\section{MIC Comparisons}

Table 1 summarizes the cumulative percentage susceptibility results for five bacterial species to increasing concentrations of piperacillin and six other beta-lactam antibiotics. These species were grouped for tabulation because of the bimodal distribution of susceptible bacterial populations. Piperacillin is the most active semisynthetic penicillin against the four Enterobacteriaceae species tested. At clinically achievable serum levels piperacillin would inhibit $74 \sim 92 \%$ of the species tested compared to $36 \sim 79 \%$ for carbenicillin or ticarcillin and $0 \sim 72 \%$ for ampicillin. Most noteworthy is the piperacillin activity versus Klebsiella pneumoniae where piperacillin inhibits $92 \%$ at $64 \mu \mathrm{g} / \mathrm{ml}$ in contrast to $36 \%$ at $128 \mu \mathrm{g} / \mathrm{ml}$ for carbenicillin/ticarcillin and $0 \%$ at $8 \mu \mathrm{g} / \mathrm{ml}$ of ampicillin. This activity is comparable to that of reference cephalosporin cephalothin $(88 \%$ at $8 \mu \mathrm{g} / \mathrm{ml})$ and second generation cephalosporins, cefamandole $(92 \%$ at $8 \mu \mathrm{g} / \mathrm{ml})$ and cefoxitin $(96 \%$ at $4 \mu \mathrm{g} / \mathrm{ml})$. The latter two compounds possess favorable in vitro characteristics as compared to currently available cephalosporin C derivatives ${ }^{1,2,3)}$. However, piperacillin also incorporates these features with broader coverage within both Enterobacter species and indole-positive Proteus species.

Species variation in susceptibility was encountered among the indole-positive Proteus species. Piperacillin, carbenicillin and ticarcillin had comparable inhibitory effects against this genus subgroup. Ampicillin and cephalothin are ineffective and cefoxitin is the most active against Pr. rettgeri and $P r$. vulgaris. Cefamandole activity would rank $P r$. rettgeri $>P r$. morganii $>P r$. vulgaris as previously reported. ${ }^{1,2,3)}$

Like the second generation cephalosporins, piperacillin had decreased activity against staphylococcal species ${ }^{1 \sim 5}$ ). All methicillin-sensitive $S$. aureus isolates were inhibited by $128 \mu \mathrm{g} / \mathrm{ml}$ of carbenicillin and ticarcillin compared to $85 \%$ at $64 \mu \mathrm{g} / \mathrm{ml}$ of piperacillin. Of note is the overlap of MICs of piperacillin and ampicillin against penicillinase-producing and deficient strains in contrast to carbenicillin and ticarcillin which show a clear bimodal distribution of the two groups. Cephalothin was most active among the cephalosporins confirming prior reports ${ }^{4,5}$.

The median MIC values for piperacillin and six other beta-lactams against five other isolates are presented in Table 2. These five bacterial species have unimodal narrow susceptibility ranges to beta-lactam antibiotics. All seven antibiotics were effective against Proteus mirabilis, Streptococcus pneumoniae and Streptococcus pyogenes. Piperacillin was the most active against Serratia species with a median MIC of $1 \mu \mathrm{g} / \mathrm{ml}$. Ampicillin and the three cephalosporins had median MICs $\geq 16 \mu \mathrm{g} / \mathrm{ml}$. Piperacillin was 8-fold more active than carbenicillin and ticarcillin against $S$. faecalis. Only ampicillin was more active (median $\mathrm{MIC}=1 \mu \mathrm{g} / \mathrm{ml}$ ) with the cephalosporins medians well above clinically achievable concentrations.

The susceptibility expressed as median MICs of non-enterobacteriaceae gram-negative bacilli are shown in Table 3. Only piperacillin was active against all Pseudomonas species tested. Piperacillin was $8 \sim 16$ times more active than ticarcillin and carbenicillin against $P$ s. aeruginosa, 128 256 times against $P_{\text {s. }}$ cepacia, 32 times against $P_{S}$. fluorescence-putida, $4 \sim 8$ times against $P$ s. stutzeri and equally inhibitory versus $P$ s. maltophilia. Ticarcillin was one $\log _{2}$ dilution more active than carbenicillin against Ps. aeruginosa confirming prior reports ${ }^{6,7)}$.

Acinetobacter anitratus isolates were only inhibited by clinically achievable concentrations of piperacillin, carbenicillin and ticarcillin. There was a marked susceptibility variation for Aeromonas 
Table 1. Cumulative percentage susceptibility of five bacterial species (135 organisms) to increasing concentrations of piperacillin and six other beta-lactam antibiotics.

\begin{tabular}{|c|c|c|c|c|c|c|c|c|c|c|c|}
\hline \multirow{2}{*}{ Organism (\#) } & \multirow{2}{*}{$\begin{array}{l}\text { Anti- } \\
\text { biotic }\end{array}$} & \multicolumn{10}{|c|}{ Cumulative $\%$ inhibited $@$ MIC $(\mu \mathrm{g} / \mathrm{ml})$ of } \\
\hline & & $\leq 0.5$ & 1 & 2 & 4 & 8 & 16 & 32 & 64 & 128 & 256 \\
\hline E. $\operatorname{coli}(29)$ & $\begin{array}{l}\text { PIPER } \\
\text { CARB } \\
\text { TICAR } \\
\text { AMPI } \\
\text { CEPHA } \\
\text { CEFAM } \\
\text { CEFOX }\end{array}$ & $\begin{array}{r}34 \\
3 \\
72\end{array}$ & $\begin{array}{r}72 \\
7 \\
17 \\
17 \\
\\
76 \\
14\end{array}$ & $\begin{array}{l}28 \\
48 \\
55 \\
10 \\
59\end{array}$ & $\begin{array}{l}79 \\
52 \\
69 \\
69 \\
41 \\
93 \\
79\end{array}$ & $\begin{array}{l}86 \\
69 \\
76 \\
72 \\
72 \\
97 \\
90\end{array}$ & $\begin{array}{r}100 \\
97\end{array}$ & $\begin{array}{l}79 \\
86\end{array}$ & $\begin{array}{r}93 \\
\\
86 \\
93 \\
100\end{array}$ & $\begin{array}{l}97 \\
90\end{array}$ & \\
\hline Enterobacter species $^{a}$ (24) & $\begin{array}{l}\text { PIPER } \\
\text { CARB } \\
\text { TICAR } \\
\text { AMPI } \\
\text { CEPHA } \\
\text { CEFAM } \\
\text { CEFOX }\end{array}$ & 17 & $\begin{array}{r}21 \\
\\
4 \\
46\end{array}$ & $\begin{array}{r}63 \\
4 \\
33 \\
\\
8 \\
67 \\
8\end{array}$ & $\begin{array}{r}75 \\
46 \\
54 \\
4 \\
\\
71 \\
17\end{array}$ & $\begin{array}{r}83 \\
54 \\
58 \\
8 \\
21 \\
83 \\
25\end{array}$ & $\begin{array}{l}88 \\
58 \\
\\
13\end{array}$ & $\begin{array}{l}63 \\
63 \\
17 \\
25 \\
88\end{array}$ & $\begin{array}{l}92 \\
71 \\
67 \\
33 \\
29\end{array}$ & $\begin{array}{l}96 \\
75 \\
71 \\
58 \\
38 \\
92 \\
33\end{array}$ & $\begin{array}{l}79 \\
83 \\
71 \\
46 \\
54\end{array}$ \\
\hline Klebsiella pneumoniae (25) & $\begin{array}{l}\text { PIPER } \\
\text { CARB } \\
\text { TICAR } \\
\text { AMPI } \\
\text { CEPHA } \\
\text { CEFAM } \\
\text { CEFOX }\end{array}$ & 40 & $\begin{array}{r}4 \\
72 \\
8\end{array}$ & $\begin{array}{l}48 \\
80 \\
72\end{array}$ & $\begin{array}{l}80 \\
88 \\
96\end{array}$ & $\begin{array}{l}88 \\
92\end{array}$ & $\begin{array}{l}84 \\
\\
24 \\
92 \\
96\end{array}$ & $\begin{array}{r}88 \\
\\
40 \\
96 \\
100\end{array}$ & $\begin{array}{r}92 \\
4 \\
20 \\
80 \\
100 \\
\\
100\end{array}$ & $\begin{array}{l}96 \\
36 \\
36 \\
84\end{array}$ & $\begin{array}{l}64 \\
64\end{array}$ \\
\hline $\begin{array}{l}\text { Proteus species }^{\mathrm{b}}(27) \\
\text { Indole-positive }\end{array}$ & $\begin{array}{l}\text { PIPER } \\
\text { CARB } \\
\text { TICAR } \\
\text { AMPI } \\
\text { CEPHA } \\
\text { CEFAM } \\
\text { CEFOX }\end{array}$ & $\begin{array}{r}52 \\
15 \\
19 \\
\\
\\
19 \\
4\end{array}$ & $\begin{array}{r}63 \\
44 \\
37 \\
4 \\
4 \\
22 \\
15\end{array}$ & $\begin{array}{r}67 \\
48 \\
41 \\
7 \\
\\
37 \\
37\end{array}$ & $\begin{array}{l}56 \\
52 \\
\\
44 \\
52\end{array}$ & $\begin{array}{l}67 \\
56 \\
11 \\
48 \\
85\end{array}$ & $\begin{array}{l}70 \\
67 \\
\\
58 \\
93\end{array}$ & $\begin{array}{l}70 \\
74 \\
\\
11 \\
74 \\
96\end{array}$ & $\begin{array}{l}74 \\
78 \\
78 \\
22 \\
15 \\
78\end{array}$ & $\begin{array}{r}48 \\
19 \\
\\
100\end{array}$ & $\begin{array}{l}85 \\
\\
81 \\
63 \\
30 \\
85\end{array}$ \\
\hline $\begin{array}{l}\text { Staphylococcus aureus (20) } \\
\text { Methicillin-sensitive }\end{array}$ & $\begin{array}{l}\text { PIPER } \\
\text { CARB } \\
\text { TICAR } \\
\text { AMPI } \\
\text { CEPHA } \\
\text { CEFAM } \\
\text { CEFOX }\end{array}$ & $\begin{array}{l}20 \\
20 \\
25 \\
25 \\
95 \\
55\end{array}$ & $\begin{array}{l}25 \\
25 \\
35 \\
90\end{array}$ & $\begin{array}{r}35 \\
\\
45 \\
100 \\
100 \\
20\end{array}$ & $\begin{array}{l}45 \\
50 \\
50 \\
55 \\
\\
\\
95\end{array}$ & $\begin{array}{l}50 \\
75 \\
80 \\
60\end{array}$ & $\begin{array}{l}60 \\
95 \\
95\end{array}$ & $\begin{array}{r}100 \\
100 \\
70\end{array}$ & 85 & $\begin{array}{r}95 \\
100\end{array}$ & 100 \\
\hline $\begin{array}{l}\text { Staphylococcus aureus (10) } \\
\text { methicillin-resistant }\end{array}$ & $\begin{array}{l}\text { PIPER } \\
\text { CARB } \\
\text { TICAR } \\
\text { AMPI } \\
\text { CEPHA } \\
\text { CEFAM } \\
\text { CEFOX }\end{array}$ & & & 30 & $\begin{array}{l}90 \\
80\end{array}$ & $\begin{array}{l}100 \\
100\end{array}$ & $\begin{array}{l}10 \\
70\end{array}$ & 100 & $\begin{array}{l}10 \\
20 \\
20 \\
30\end{array}$ & $\begin{array}{r}90 \\
90 \\
100\end{array}$ & $\begin{array}{r}50 \\
100 \\
100\end{array}$ \\
\hline
\end{tabular}

a. Includes Enterobacter cloacae (10), Enterobacter aerogenes (8) and Enterobacter agglomerans (6).

b. Includes Proteus morganii (12), Proteus rettgeri (9), Proteus vulgaris (6).

hydrophila to the seven antimicrobics with cefamandole and piperacillin being most active.

Among the 108 PS. aeruginosa isolates tested, several strains were resistant ( $\geq 8 \mu \mathrm{g} / \mathrm{ml})$ to gentamicin and/or tobramycin. These 14 isolates and the sensitive strains of Ps. aeruginosa are shown in Table 4. Increased resistance to the aminoglycosides was accompanied by increased resistance to the semisynthetic penicillins e.g. piperacillin, carbenicillin and ticarcillin. Only the piperacillin MICs remained at or below clinically achievable concentrations for these gentamicin resistant and gentamicintobramycin resistant strains.

\section{MIC-MLC Comparisons}

The MLC/MIC ratios for piperacillin, ticarcillin and carbenicillin are shown in Table 5. Only 2 
Table 2. Median MIC concentrations $(\mu \mathrm{g} / \mathrm{ml})$ of selected bacterial species having unimodal population distributions to piperacillin and six other beta-lactam antibiotics.

\begin{tabular}{l|c|c|c|c|c}
\hline \multirow{2}{*}{ Antibiotic } & $\begin{array}{c}\text { Proteus } \\
\text { mirabilis } \\
(24)^{\mathrm{a}}\end{array}$ & $\begin{array}{c}\text { Serratia } \\
\text { species } \\
(20)\end{array}$ & $\begin{array}{c}|c| \\
\text { faecalis } \\
(15)\end{array}$ & $\begin{array}{c}\text { Sneumoniae } \\
(20)\end{array}$ & $\begin{array}{c}\text { pyogenes } \\
(20)\end{array}$ \\
\hline Piperacillin & $\leq 0.5$ & 1.0 & 4.0 & $\leq 0.25$ & $\leq 0.25$ \\
Carbenicillin & $\leq 0.5$ & 4.0 & 32 & $\leq 0.25$ & $\leq 0.25$ \\
Ticarcillin & $\leq 0.5$ & 4.0 & 32 & $\leq 0.25$ & $\leq 0.25$ \\
Ampicillin & 1.0 & 32 & 1.0 & $\leq 0.25$ & $\leq 0.25$ \\
Cephalothin & 2.0 & $>256$ & 32 & $\leq 0.25$ & $\leq 0.25$ \\
Cefamandole & $\leq 0.5$ & 32 & 32 & $\leq 0.25$ & $\leq 0.25$ \\
Cefoxitin & 2.0 & 16 & $>256$ & 1.0 & 0.5 \\
\hline
\end{tabular}

a. Number of isolates tested.

Table 3. Median MIC concentrations $(\mu \mathrm{g} / \mathrm{ml})$ for non-Enterobacteriaceae gram-negative bacilli including 138 isolates of Pseudomonas species to piperacillin and six other beta-lactam antibiotics.

\begin{tabular}{|c|c|c|c|c|c|c|c|}
\hline \multirow[b]{2}{*}{ Antibiotic } & \multicolumn{5}{|c|}{ Pseudomonas } & \multirow{2}{*}{$\begin{array}{c}\text { Acinetobacter } \\
\text { anitratus } \\
\text { (11) }\end{array}$} & \multirow{2}{*}{$\begin{array}{c}\text { Aeromonas } \\
\text { hydrophila } \\
\text { (11) }\end{array}$} \\
\hline & $\begin{array}{c}\text { aeruginosa } \\
(108)^{\mathrm{a}}\end{array}$ & $\begin{array}{c}\text { cepacia } \\
\text { (10) }\end{array}$ & $\begin{array}{c}\text { Aluorescens- } \\
\text { putida } \\
\text { (6) }\end{array}$ & $\begin{array}{c}\text { maltophilia } \\
\text { (10) }\end{array}$ & $\begin{array}{l}\text { stutzeri } \\
\text { (4) }\end{array}$ & & \\
\hline Piperacillin & 2.0 & 1.0 & 8.0 & 8.0 & $\leq 0.5$ & 8.0 & 2.0 \\
\hline Carbenicillin & 32 & 256 & $>256$ & 8.0 & 4.0 & 8.0 & 256 \\
\hline Ticarcillin & 16 & 128 & 256 & 8.0 & 2.0 & 4.0 & 128 \\
\hline Ampicillin & $>256$ & $>256$ & 128 & $>256$ & $\leq 0.5$ & 16 & $>256$ \\
\hline Cephalothin & $>256$ & $>256$ & $>256$ & $>256$ & 256 & 256 & 256 \\
\hline Cefamandole & $>256$ & $>256$ & $>256$ & 64 & 32 & 16 & 1.0 \\
\hline Cefoxitin & $>256$ & 64 & $>256$ & 64 & 8.0 & 32 & 8.0 \\
\hline
\end{tabular}

a. Number of isolates tested

Table 4. Relationship of piperacillin, carbenicillin and ticarcillin MIC values in Pseudomonas aeruginosa isolates having aminoglycoside resistance.

\begin{tabular}{c|c|c|c|c}
\hline Aminoglycoside susceptibility & \multirow{2}{*}{$\begin{array}{c}\text { No. } \\
\text { Gentamicin / Tobramycin }\end{array}$} & \multicolumn{3}{|c}{ Median(range) MIC in $\mu \mathrm{g} / \mathrm{ml} ;$} \\
\cline { 5 - 5 } & & Pested & Ticarcillin & Carbenicillin \\
\hline Sensitive / Sensitive & 94 & $2.0(\leq 0.5 \sim 128)$ & $16(\leq 0.5 \sim>256)$ & $32(\leq 0.5 \sim>256)$ \\
Resistant / Sensitive & 6 & $32(8 \sim 64)$ & $256(64 \sim>256)$ & $256(128 \sim>256)$ \\
Resistant / Resistant & 8 & $64(16 \sim 256)$ & $>256(128 \sim>256)$ & $>256(256 \sim>256)$ \\
\hline
\end{tabular}

a. Sensitive is $=$ or less than $4 \mu \mathrm{g} / \mathrm{ml}$ to both aminoglycosides tested in cation supplement MUELLERHINTON broth.

of 79 organisms $(3 \%)$ had MLC values more than 8 -fold higher than the piperacillin MIC. These included single strains of Proteus morganii and Pseudomonas aeruginosa. Ticarcillin had the most with 3/79 and carbenicillin the least with 1/79. MLC/MIC ratios were also determined for ampicillin, cephalothin, cefamandole and cefoxitin. Cefamandole demonstrated the widest variation between inhibitory and cidal endpoints confirming previous reports ${ }^{1,2}$. Seventeen percent of MLCs were $>8$ fold greater than cefamandole MICs. Nearly all of these were Enterobacter species, indole-positive Proteus species and Serratia. Cefoxitin had ratios similar to piperacillin and superior to other cephalosporins $^{1,2)}$.

\section{Inoculum Size MIC-MLC Comparisons}

The effects of inoculum size on MIC and MLC results were studied on 79 organisms at $10^{3}, 10^{5}$, 
Table 5. MIC-MLC comparison of piperacillin, carbenicillin and ticarcillin for 79 organisms @ $10^{5} \mathrm{CFU} / \mathrm{ml}$ inoculum size.

\begin{tabular}{|c|c|c|c|c|c|}
\hline \multirow{2}{*}{ Organism (\#) } & \multirow{2}{*}{ Antibiotic } & \multicolumn{4}{|c|}{ MLC/MIC ratios } \\
\hline & & 1 & 2 & 4 & 8 or more \\
\hline E. coli $(10)$ & $\begin{array}{l}\text { Piperacillin } \\
\text { Ticarcillin } \\
\text { Carbenicillin }\end{array}$ & $\begin{array}{l}8 \\
8 \\
8\end{array}$ & $\begin{array}{l}1 \\
1 \\
2\end{array}$ & $\begin{array}{l}1 \\
1 \\
0\end{array}$ & $\begin{array}{l}0 \\
0 \\
0\end{array}$ \\
\hline Klebsiella pneumoniae (10) & $\begin{array}{l}\text { Piperacillin } \\
\text { Ticarcillin } \\
\text { Carbenicillin }\end{array}$ & $\begin{array}{l}8 \\
8 \\
5\end{array}$ & $\begin{array}{l}2 \\
2 \\
5\end{array}$ & $\begin{array}{l}0 \\
0 \\
0\end{array}$ & $\begin{array}{l}0 \\
0 \\
0\end{array}$ \\
\hline Enterobacter species $^{a}(10)$ & $\begin{array}{l}\text { Piperacillin } \\
\text { Ticarcillin } \\
\text { Carbenicillin }\end{array}$ & $\begin{array}{r}8 \\
10 \\
4\end{array}$ & $\begin{array}{l}1 \\
0 \\
5\end{array}$ & $\begin{array}{l}1 \\
0 \\
1\end{array}$ & $\begin{array}{l}0 \\
0 \\
0\end{array}$ \\
\hline Proteus mirabilis (10) & $\begin{array}{l}\text { Piperacillin } \\
\text { Ticarcillin } \\
\text { Carbenicillin }\end{array}$ & $\begin{array}{l}10 \\
10 \\
10\end{array}$ & $\begin{array}{l}0 \\
0 \\
0\end{array}$ & $\begin{array}{l}0 \\
0 \\
0\end{array}$ & $\begin{array}{l}0 \\
0 \\
0\end{array}$ \\
\hline Indole-positive Proteus species ${ }^{b}(10)$ & $\begin{array}{l}\text { Piperacillin } \\
\text { Ticarcillin } \\
\text { Carbenicillin }\end{array}$ & $\begin{array}{l}7 \\
6 \\
5\end{array}$ & $\begin{array}{l}1 \\
2 \\
4\end{array}$ & $\begin{array}{l}1 \\
1 \\
0\end{array}$ & $\begin{array}{l}1 \\
1 \\
1\end{array}$ \\
\hline Serratia marcescens (10) & $\begin{array}{l}\text { Piperacillin } \\
\text { Ticarcillin } \\
\text { Carbenicillin }\end{array}$ & $\begin{array}{l}6 \\
5 \\
4\end{array}$ & $\begin{array}{l}3 \\
1 \\
3\end{array}$ & $\begin{array}{l}1 \\
3 \\
3\end{array}$ & $\begin{array}{l}0 \\
1 \\
0\end{array}$ \\
\hline Pseudomonas aeruginosa (9) & $\begin{array}{l}\text { Piperacillin } \\
\text { Ticarcillin } \\
\text { Carbenicillin }\end{array}$ & $\begin{array}{l}2 \\
4 \\
1\end{array}$ & $\begin{array}{l}3 \\
3 \\
4\end{array}$ & $\begin{array}{l}3 \\
1 \\
4\end{array}$ & $\begin{array}{l}1 \\
1 \\
0\end{array}$ \\
\hline Staphylococcus aureus (10) & $\begin{array}{l}\text { Piperacillin } \\
\text { Ticarcillin } \\
\text { Carbenicillin }\end{array}$ & $\begin{array}{r}6 \\
9 \\
10\end{array}$ & $\begin{array}{l}3 \\
0 \\
0\end{array}$ & $\begin{array}{l}1 \\
1 \\
0\end{array}$ & $\begin{array}{l}0 \\
0 \\
0\end{array}$ \\
\hline
\end{tabular}

a. Includes five strains each of Ent. aerogenes and Ent. cloacae.

b. Includes Proteus morganii (5), Proteus rettgeri (3) and Proteus vulgaris (2).

and $10^{7}$ colony forming units $/ \mathrm{ml}$ (Table 6 ). Only those organisms (listed in Table 5) not having off-scale MIC values were tabulated. Lower numbers of cefamandole MIC and MLC were noted due to a large incidence of $\leq 0.5 \mu \mathrm{g} / \mathrm{ml}$ MIC-MLC values among the Enterobacteriaceae in addition to $>256 \mu \mathrm{g} / \mathrm{ml}$ MIC-MLC against $P S$. aeruginosa, Serratia and some Proteus species.

Ticarcillin, carbenicillin and cefoxitin showed minimal variation (increase) in MIC or MLC when the inoculum was raised from $10^{3}$ to $10^{5}$ $\mathrm{CFU} / \mathrm{ml}$ and $10^{5}$ to $10^{7} \mathrm{CFU} / \mathrm{ml}$. Ninety-two to $100 \%$ of the endpoints were $\leq 3 \log _{2}$ dilutions. Piperacillin was similar to the above agents at $10^{3} \sim 10^{5} \mathrm{CFU} / \mathrm{ml}$, but had $24 \sim 25 \%$ of MICMLC results markedly altered ( $>8$-fold increase) by inoculum increases to $10^{7} \mathrm{CFU} / \mathrm{ml}$. Nearly all isolates having piperacillin susceptibility inoculum effect were Klebsiella pneumoniae (mean MICMLC increase $=64$-fold). Cefamandole MICs and MLCs were markedly increased by raising inoculum
Table 6. Percent of isolates having MIC or MLC results within $3 \log _{2}$ dilutions ( $\leq 8$ fold increase) after increasing the inoculum concentration $10^{2}$ $\mathrm{CFU} / \mathrm{ml}$ for piperacillin, ticarcillin, carbenicillin, cefamandole and cefoxitin.

\begin{tabular}{|c|c|c|c|c|}
\hline \multirow[t]{2}{*}{ Antibiotic (\#) } & \multicolumn{2}{|c|}{$\begin{array}{l}10^{3} \sim 10^{5} \\
\mathrm{CFU} / \mathrm{ml}^{\mathrm{a}}\end{array}$} & \multicolumn{2}{|c|}{$\begin{array}{l}10^{5} \sim 10^{7} \\
\text { CFU } / \mathrm{ml}^{\mathrm{b}}\end{array}$} \\
\hline & MIC & MLC & MIC & MLC \\
\hline Piperacillin (48) & 94 & 97 & 76 & 75 \\
\hline Ticarcillin (51) & 98 & 94 & 92 & 92 \\
\hline $\begin{array}{l}\text { Carbenicillin } \\
(54)\end{array}$ & 100 & 98 & 96 & 98 \\
\hline $\begin{array}{l}\text { Cefamandole } \\
\text { (24) }\end{array}$ & 100 & 62 & 45 & 63 \\
\hline Cefoxitin (68) & 98 & 95 & 96 & 96 \\
\hline
\end{tabular}

a. Comparison of MIC and MLC results at $10^{5}$ $\mathrm{CFU} / \mathrm{ml}$ inoculum concentration compared to those at $10^{3} \mathrm{CFU} / \mathrm{ml}$.

b. Comparison of MIC and MLC results at $10^{7}$ $\mathrm{CFU} / \mathrm{ml}$ inoculum concentration compared to those at $10^{5} \mathrm{CFU} / \mathrm{ml}$. 
concentrations. From 38 to $55 \%$ of the strains tested had cefamandole MIC-MLCs showing increases of $>8$-fold with several isolates MLC 32- to 128-fold higher at $10^{7} \mathrm{CFU} / \mathrm{ml}$.

\section{Discussion}

Piperacillin (T-1220) belongs to the semisynthetic group of penicillins and is structurally similar to ampicillin. More extensive $\alpha$-substitutions have provided this compound with a broad antimicrobial spectrum that includes gram-positive and gram-negative bacteria, especially Pseudomonas, Klebsiella, Serratia, Proteus and Enterococci. The compound is poorly absorbed orally, but high serum concentrations are achieved by parenteral routes.

Other new semisynthetic penicillins that most resemble piperacillin are BL-P1654 (Bristol), PC904 (Sumitomo) and pirbenicillin (CP-33,994-2, Pfizer). All of these antimicrobics have antipseudomonas activity 8 - to 16 -fold that of carbenicillin $\left.{ }^{8} 10\right)$. However, each has one or more disadvantages when compared to piperacillin. BL-P1654 is adversely affected by both serum and increased inoculum concentrations, has a limited spectrum versus all Proteus species and lastly is toxic to some animal species $^{11)}$. PC-904 also is less active than piperacillin against Proteus mirabilis, the indole-positive Proteus species and Serratia species ${ }^{9}$. Like PC-904, pirbenicillin has little activity on Proteus species and is significantly more protein bound than piperacillin ${ }^{10)}$. Piperacillin compared to carbenicillin and ticarcillin had a wider antimicrobial spectrum and increased activity, particularly against Pseudomonas species, Serratia and Klebsiella pneumoniae. Like the new cephalosporins, piperacillin showed excellent inhibitory activity against Enterobacter species and indole-positive Proteus species, while adding the Enterococci, Serratia species, and K. pneumoniae to its spectrum. Only ampicillin was superior to piperacillin against $S$. faecalis.

The only apparent drawbacks to piperacillin were the slight inoculum size effects on MIC and MLCs at $10^{7} \mathrm{CFU} / \mathrm{ml}$ levels, particularly among $\mathrm{K}$. pneumoniae isolates. Also piperacillin is susceptible to beta-lactamase activity similar to carbenicillin, ticarcillin and ampicillin. These features may limit its efficacy against beta-lactamase-producing $S$. aureus, H. influenzae, $N$. gonorrhoeae and some populations of Enterobacteriaceae including E. coli.

The combination of excellent in vitro inhibiting effects on a wide spectrum of bacteria and in vivo pharmacology makes piperacillin (T-1220) a very promising parenteral antimicrobic. If dosages were used permitting piperacillin serum levels of $64 \mu \mathrm{g} / \mathrm{ml}$, this would inhibit $96 \%$ of the gram-positive and negative organisms in a clinical isolate study of 10,858 strains $^{12}$. Ninety-one percent would be covered by concentrations of $16 \mu \mathrm{g} / \mathrm{ml}$. These in vitro data coupled with a $22 \%$ serum protein binding, active urinary excretion $(66 \%)$, high biliary concentrations, favorable human pharmacokinetics, and effective treatment of experimental animal infection favors further human in vivo investigations. Additional in vitro studies on anaerobic bacteria are also needed.

\section{Acknowledgements}

We gratefully acknowledge the expert technical assistance of CAROLYN BAKER, ROLLIE PACKER and RoBERT BADAl. Also we thank Barbara Beardsley for her help in the preparation of this manuscript.

\section{References}

1) Barry, A. L.; C. Thornsberry, R. N. Jones, P. C. Fuchs, T. L. Gavan \& E. H. Gerlach: Cefuroxime, in vitro comparison to six other parenteral cephalosporins. (in press), 1977

2) Jones, R. N.; C. Thornsberry, A. L. Barry, P. C. Fuchs, T. L. Gavan \& E. H. Gerlach: BL-S786, a new parenteral cephalosporin. II. In vitro antimicrobial activity comparison with six related cephalosporins. J. Antibiotics 30: 583 592, 1977

3) Jones, R. N. \& P. C. Fuchs: Comparison of in vitro antimicrobial activity of cefamandole and cefazolin with cephalothin against over 8,000 clinical bacterial isolates. Antimicr. Agents \& Chemoth. 9: 1066 1069,1976

4) Jones, R. N.; P. C. Fuchs, T. L. Gavan, E. H. Gerlach, A. L. Barry \& C. Thornsberry: BL-S786, 
a new parenteral cephalosporin. I. A collaborative in vitro susceptibility comparison to cephalothin against 5,762 clinical bacterial isolates. J. Antibiotics 30: 576 582, 1977

5) Jones, R. N.; P. C. Fuchs, T. L. Gavan, E. H. Gerlach, A. L. Barry \& C. Thornsberry: Cefuroxime, a new parenteral cephalosporin: Collaborative in vitro susceptibility comparison with cephalothin against 5,887 clinical bacterial isolates. Antimicr. Agents \& Chemoth. 12: 47 50, 1977

6) Fuchs, P. C.; T. L. Gavan, E. H. Gerlach, R. N. Jones, A. L. Barry \& C. Thornsberry: Ticarcillin: a collaborative in vitro comparison with carbenicillin against over 9,000 clinical bacterial isolates. Am. J. Med. Sci. (in press). 1977

7) Fuchs, P. C; C. Thornsberry, A. L. Barry, T. L. Gavan, E. H. Gerlach \& R. N. Jones: Ticarcillin, carbenicillin and BL-P1908: In vitro comparison of three antipseudomonal semisynthetic penicillins. J. Antibiotics 30: 1106 1114, 1977

8) Price, K. E. ; F. Leitner, M. Misiek, D. R. Chisholm \& T. A. Pursiano: BL-P1654, a new broad spectrum penicillin. Antimicr. Agents \& Chemoth. -1970: 17 29, 1971

9) Noguchi, H.; Y. Eda, H. Tobiki, T. Nakagome \& T. Komatsu: PC-904, a novel broad-spectrum semisynthetic penicillin with marked antipseudomonal activity: Microbiological evaluation. Antimicr. Agents \& Chemoth. 9: 262 273, 1976

10) Wise, R.; J.M.Andrews \& K.A. Bedord: Pirbenicillin, a semisynthetic penicillin with antipseudomonal activity. J. Antimicr. Chemoth. 3: $175 \sim 183,1977$

11) SCOY, R. E. V.; E. WARREN \& J. A. WASHINGTON: In vitro antimicrobial activity of a new semisynthetic penicillin BL-P1654. Antimicr. Agents \& Chemoth. -1970: 12 16, 1971

12) Gerlach, E. H.; P. C. Fuchs, R. N. Jones, T. L. Gavan, A. L. Barry \& C. Thornsberry: Piperacillin, a new parenteral broad spectrum penicillin: Collaborative in vitro susceptibility comparison with carbenicillin against 10,858 clinical bacterial isolates. Antimicr. Agents \& Chemoth. (in press), 1978 